\title{
Retrobulbar oedema after ophthalmic plaque radiotherapy
}

\author{
Paul T Finger, Sandra Frazier Byrne, Darius M Moshfeghi, Henry D Perry
}

Retrobulbar oedema has been associated with posterior scleritis and uveal melanoma following ophthalmic plaque radiotherapy. ${ }^{1}$ We have noted massive retrobulbar oedema after episcleral plaque radiotherapy in a patient with a medium sized choroidal melanoma. ${ }^{23}$ The diagnosis of retrobulbar oedema was supported by the echographic finding of a completely echolucent lesion on B-scan which partially resolved over time. Our echographic diagnosis of retrobulbar oedema was sustained when continued tumour enlargement brought this eye to histopathological evaluation.

\section{Case report}

A 69-year-old man was treated with iodine-125 plaque radiotherapy together with adjuvant microwave thermotherapy for his choroidal melanoma. ${ }^{2}$ Before treatment the tumour's dimensions were noted to be $10 \times 10 \mathrm{~mm}$ (base) and $6.4 \mathrm{~mm}$ (apex). The tumour received $55 \mathrm{~Gy}$ to the apex, while the lesion's base (calculated as the inner scleral point, $1 \mathrm{~mm}$ along the central axis of the plaque) received $292 \mathrm{~Gy}$. Microwave

\section{Department of \\ Ophthalmology, Ocular \\ Tumor Service, The New \\ York Eye and Ear \\ Infirmary, New York \\ City, USA and the \\ Department of \\ Ophthalmology, Division \\ of Ocular Tumor and \\ Orbital Disease, North \\ Shore University \\ Hospital, Cornell \\ University Medical \\ College, Manhasset, New \\ York, USA \\ $P$ T Finger \\ H D Perry \\ D M Moshfeghi}

The Bascom Palmer Eye Institute, Department of Ophthalmology,

University of Miami

Medical School, Miami,

Florida, USA

S F Byrne

Tulane Medical School, New Orleans, Louisiana, USA

D M Moshfeghi

Correspondence to:

Paul T Finger, MD, The Ocular Tumor Service, The New York Eye and Ear New York Eye and Ear New York, NY 10003, USA

Accepted for publication 7 May 1993
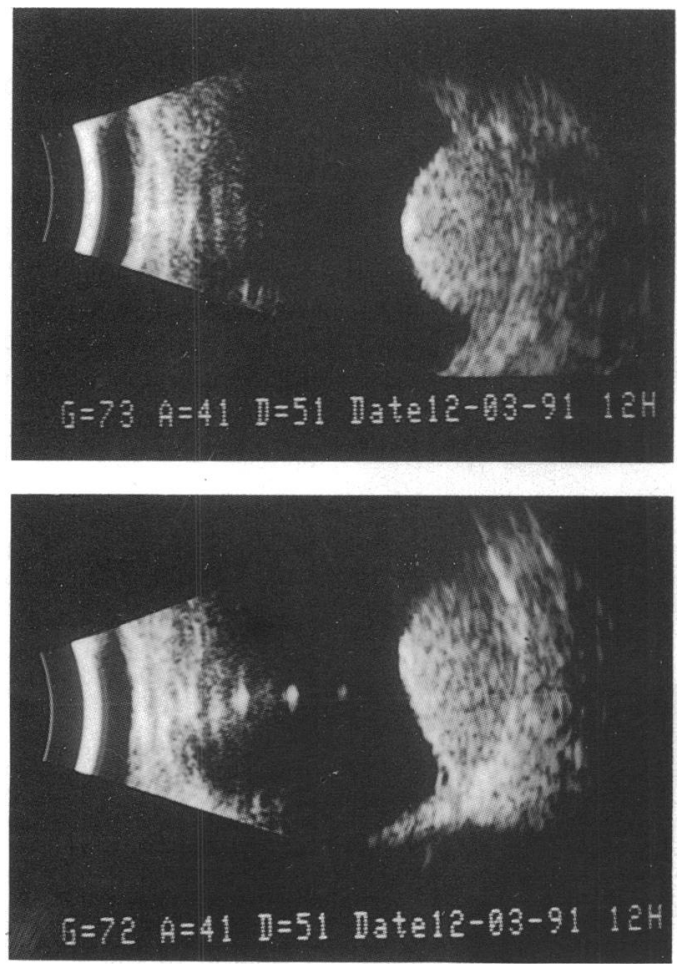

Figure 1 Initial echography examination. Transverse (top) and longitudinal (bottom) B-scan echograms show a large orbital fat adjacent to the tumour indicating an absence of extrascleral extension. The maximal height of the tumour measured on A-scan was $7 \cdot 4 \mathrm{~mm}$ dome shaped tumour. Note the normal appearance of the hyperthermia $\left(47 \cdot 3^{\circ} \mathrm{C}\right.$ for 45 minutes to the episclera) was used to augment the effect of radiotherapy. ${ }^{245}$

Twenty three months after treatment ophthalmoscopy revealed an increase in the tumour's basal dimension to $16 \times 13 \mathrm{~mm}$, while echography revealed an apical tumour height of $7 \cdot 4 \mathrm{~mm}$ (Fig 1). Our patient elected to be treated with the lower energy palladium-103 plaque radiotherapy rather than lose his eye. ${ }^{3}$ At that time the tumour received an apex dose of $81 \mathrm{~Gy}$ and an inner scleral dose of $361 \mathrm{~Gy}$. By the end of his second treatment, the patient's sclera (beneath the tumour) had received a cumulative radiation dose of $653 \mathrm{~Gy}$.

Two months after palladium-103 radiotherapy, a standardised A-scan revealed an apical tumour height of $9.6 \mathrm{~mm}$. B-scan demonstrated a massive $(15 \times 11 \times 2.3 \mathrm{~mm})$ completely echolucent lesion in the orbit adjacent to the intraocular tumour (Fig 2). No internal reflectivity could be demonstrated at high gain settings. Six months after treatment, B-scan revealed a
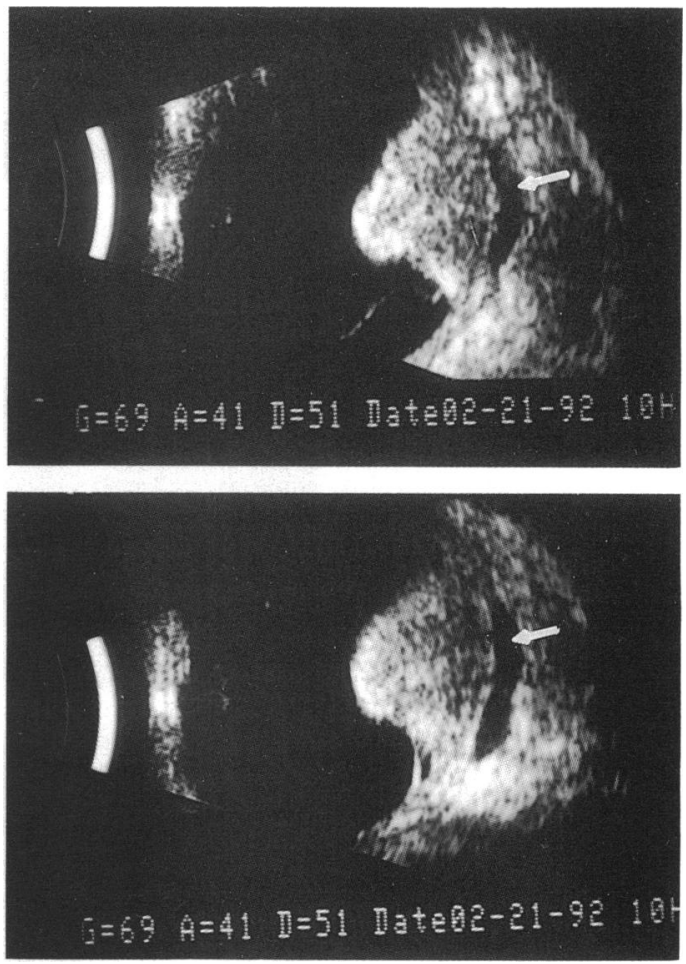

Figure 2 Three month postoperative examination.

Transverse (top) and longitudinal (bottom). B-scan echograms show the diffuse, well circumscribed, echolucent lesion in the episcleral space adjacent to the tumour (arrows), presumably due to oedema in Tenon's space. B-scan echograms also show that the tumour has increased in size. A-scan measurements indicated that the tumour had increased in height from $7.4 \mathrm{~mm}$ preoperatively to $9.6 \mathrm{~mm}$ at this examination. 


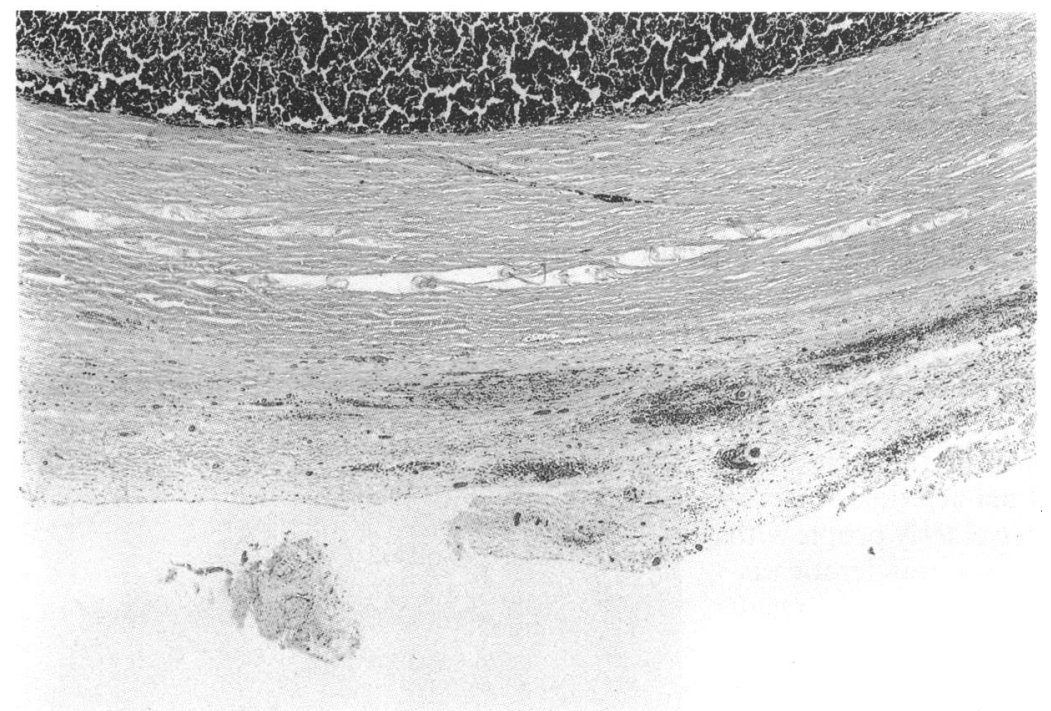

Figure 3 Light microscopy of the enucleated eye demonstrated that the sclera beneath the tumour was thickened. A plaque of episcleral tissue containing pigmented macrophages was also noted (haematoxylin and eosin, $\times 23$ ). decrease in the size of the echolucent retrobulbar lesion (confirming the diagnosis of retrobulbar oedema). Then, owing to documented tumour enlargement to an apical height of $10.4 \mathrm{~mm}$, our patient underwent enucleation of his left eye.

Histopathological evaluation revealed decreased tumour viability as shown by the predominance of necrotic cells. The sclera beneath the tumour was thickened with focal areas of oedema and a $0.5 \mathrm{~mm}$ (thick) plaque of episcleral pigmented tissue (Fig 3). The pigmented cells were mostly macrophages containing haemosiderin. A Prussian blue stain for iron confirmed the presence of haemosiderin. The plaque of episcleral tissue was contiguous with two areas of extrascleral oedema and haemorrhage. There was no extrascleral extension of the choroidal melanoma.

\section{Comment}

This case illustrates that scleral oedema and/or thickening after plaque radiotherapy can be associated with ultrasonic evidence of retrobulbar oedema. ${ }^{67}$ Although this finding could be confused with extrascleral tumour extension, there are several echographic features that aided in the differentiation. With high resolution Bscan (and a high gain setting), oedema fluid produces no echoes resulting in a completely echolucent pattern. In contrast, tumour extension usually produces internal echoes from clumps of tumour cells and blood vessels (acoustic interfaces). Another finding in retrobulbar oedema is a diffuse, smooth appearance as opposed to the more nodular configuration of tumour extending into orbit. Gentle compression testing can show the oedema to be soft (compressible), whereas tumour tissue is relatively hard (non-compressible). A-scan can also be used to assess the internal reflectivity and vascularity of an epibulbar lesion. Whereas melanomatous extension produces low to medium reflectivity and a vascular pattern, oedema is extremely low, reflective, and nonvascular.

The epibulbar inflammatory tissue that may accompany an irradiated uveal melanoma can also be differentiated from true tumour extension. Although this tissue is solid and may be low to medium reflective (as in uveal melanoma), it is normally more diffuse, nonvascular, and either disappears or remains the same size over time. Extrascleral tumour extension tends to enlarge progressively.

Retrobulbar oedema could also be confused with a localised episcleral haematoma. We have found that haematomas are absorbed relatively quickly, such that echographic evidence of haemorrhage will tend to persist for days to weeks rather than last for several months as noted with oedema. Lastly, as localised haemorrhages reabsorb they will demonstrate changes in internal reflectivity over time.

1 Byrne SF, Green RL. Intraocular tumors. In: Ultrasound of the eye and orbit. St Louis: Mosby 1992: 133-211.

2 Finger PT. Microwave plaque thermoradiotherapy for choroidal melanoma. Brf Ophthalmol 1992; 76: 358-64

3 Finger PT, Moshfeghi DM, Ho TK. Palladium-103 ophthalmic plaque radiotherapy. Arch Ophthalmol 1991; 109: 1610-3.

$4 \mathrm{Kim}$ JH, Hahn EW, Ahmed SA. Combination hyperthermi and radiation therapy for malignant melanoma. Cancer 1982; 50: 478-82.

5 Manning MR, Cetas TC, Miller RC, Oleson JR, Conner WG, Gerner EW. Clinical hyperthermia: results of a phase I trial employing hyperthermia alone or in combination with external beam or interstitial radiotherapy. Cancer 1982; 49: 205-16.

6 Finger PT, Curtin BJ, Packer S, Svitra PP, Iwamoto T, Whitmore WG, et al. Scleral hyperplasia induced by heat. Am F Ophthalmol 1986; 102: 25-32.

7 Finger PT, Perry HD, Packer S, Erdey RA, Weisman GD, Sibony PA. Posterior scleritis as an intraocular tumor. Br f Ophthalmol 1990; 74: 121-2. 